Tohoku Math. J.

56 (2004), 423-443

\title{
EXAMPLES OF LORENTZIAN GEODESIBLE FOLIATIONS OF CLOSED THREE-MANIFOLDS HAVING HEEGAARD SPLITTINGS OF GENUS ONE
}

\author{
Ken YoKUMOTO
}

(Received February 5, 2003, revised August 27, 2003)

\begin{abstract}
We construct codimension-one, Lorentzian geodesible foliations of closed three-manifolds having Heegaard splittings of genus one. We prove that all the inner leaves of a Reeb component of a codimension-one, totally geodesic foliation of a Lorentzian threemanifold are spacelike, and the boundary leaf of a Reeb component is lightlike.
\end{abstract}

1. Introduction. Geodesibility of a given codimension-1 foliation $\mathcal{F}$ has been studied by several authors. Here geodesibility of $\mathcal{F}$ means that $\mathcal{F}$ is totally geodesic for some complete Riemannian metric, and in this case $\mathcal{F}$ is called complete Riemannian geodesible. Let us first recall a couple of results about a codimension-1, totally geodesic foliation $\mathcal{F}$ of a complete Riemannian manifold $(M, g)$. Blumenthal and Hebda ([1]) showed that the universal covering of $M$ is a product $L \times \boldsymbol{R}$ and the lift of $\mathcal{F}$ is the product foliation $\{L \times\{*\}\}$, where $L$ is the universal covering of the leaves of $\mathcal{F}$. Oshikiri ([9], [10]) proved that any Killing field with bounded length preserves $\mathcal{F}$. Regarding codimension-1, complete Riemannian geodesible foliations, we remark the following results. Carrière and Ghys ([3]) classified a codimension-1, complete Riemannian geodesible foliation of a closed 3-manifold. Ghys ([5]) classified a codimension-1, complete Riemannian geodesible foliation of a closed manifold. Thus a codimension-1, complete Riemannian geodesible foliation of a closed manifold is well understood.

Now we consider codimension-1, Lorentzian geodesible foliations. Zeghib ([14]) constructed codimension-1, lightlike totally geodesic foliations. He constructed a lightlike totally geodesic foliation $\mathcal{F}$ in the following cases:

(1) $\mathcal{F}$ is defined by a locally free action with codimension- 1 orbits of a Lie group with a 1-dimensional normal subgroup.

(2) $\mathcal{F}$ is the suspension of a foliation $\mathcal{L}$ of a Riemannian manifold $(M, g)$ by a diffeomorphism of $M$ preserving $\mathcal{L}$ and $\left.g\right|_{T \mathcal{L}}$.

Although this construction gives an example of a codimension-1, Lorentzian geodesible foliation, we do not know whether it is complete Lorentzian geodesible or not (for example, consider a non-singular flow on a 2-torus with Reeb components and the following). Carrière and Rozoy ([4]) proved that the canonical lightlike totally geodesic foliations of a lightlike

2000 Mathematics Subject Classification. Primary 57R30; Secondary 53C50.

Key words and phrases. Lorentzian manifolds, totally geodesic foliations. 
complete Lorentzian 2-torus are $C^{0}$-linearizable. So there is a difference between complete Lorentzian geodesible foliations and Lorentzian geodesible ones.

By the theorem of Blumenthal and Hebda ([1]), there exist no codimension-1, Riemannian geodesible foliations of a closed manifold with finite fundamental group. We have the following question.

QUESTION 1.1. Does there exist a codimension-1, Lorentzian geodesible foliation of a closed manifold with finite fundamental group?

Although a Riemannian metric on a closed manifold is always complete, a Lorentzian metric on a closed manifold is not always complete. Hence it is a serious matter whether to suppose completeness of Lorentzian metrics when we consider totally geodesic foliations of closed manifolds. Recall that a geodesic of an incomplete Lorentzian metric on a closed manifold has an infinite Riemannian length whenever it is not closed. Thus if we consider, for example, an Ehresmann connection for a foliation ([2]) of a closed manifold, then it seems that the assumption of completeness of the Lorentzian metric is unnecessary. Therefore it seems meaningful to consider totally geodesic foliations of closed Lorentzian manifolds without assuming completeness. Hence, first of all, we consider codimension-1, Lorentzian geodesible foliations. However, completeness must be important. Actually, the present author proved the following (see [12]): There exists no totally geodesic foliation of a lightlike complete Lorentzian 2-torus which contains at least two kinds of leaves among spacelike, timelike, and lightlike ones. There is an example of a totally geodesic foliation of a lightlike incomplete Lorentzian 2-torus which contains at least two kinds of leaves. We have a partial answer to Question 1.1 as follows.

THEOREM 3.1. If a closed 3-manifold has a Heegaard splitting of genus one, then it has a codimension-1 Lorentzian geodesible foliation.

We prove this theorem by constructing examples in Section 3.2. Each example has spacelike and lightlike leaves. So the following question arises.

QUESTION 1.2. Does a codimension-1, totally geodesic foliation of a closed Lorentzian manifold with finite fundamental group have lightlike leaves?

In Section 4, we consider totally geodesic foliations consisting of spacelike leaves of compact Lorentzian manifold, and codimension-1, totally geodesic foliations consisting of timelike leaves of Lorentzian 3-manifold. We do not assume completeness of Lorentzian metrics. We then have the following.

PROPOSITION 4.2. Let $(M, g)$ be a compact Lorentzian manifold and $\mathcal{F}$ a totally geodesic foliation of $M$ consisting of spacelike leaves. Assume that $\mathcal{F}$ is tangent to $\partial M$ and $(T \mathcal{F})^{\perp}$ is completely integrable. Then the distribution $(T \mathcal{F})^{\perp}$ is an Ehresmann connection for $\mathcal{F}$. 
Proposition 4.4. Let $M$ be an orientable 3-manifold, $g$ a time-orientable Lorentzian metric on $M$, and $\mathcal{F}$ an orientable, codimension-1, totally geodesic foliation of $M$ consisting of timelike leaves. Denote the foliation determined by $(T \mathcal{F})^{\perp}$ by $\mathcal{H}$. Then the following hold.

(1) The lightlike vectors on $T \mathcal{F}$ determine two subfoliations $\mathcal{L}_{0}, \mathcal{L}_{1}$ of $\mathcal{F}$.

(2) The distribution $T \mathcal{H} \oplus T \mathcal{L}_{i}$ is completely integrable for $i=0,1$. Therefore, if $\mathcal{H}_{i}$ denotes the foliation determined by $T \mathcal{H} \oplus T \mathcal{L}_{i}, i=0,1$, then $\left(\mathcal{F}, \mathcal{H}_{0}, \mathcal{H}_{1}\right)$ is a total foliation of $M$ (We call it the total foliation associated with $\mathcal{F}$ ).

In Section 5, we consider a Reeb component of a codimension-1, totally geodesic foliation of a Lorentzian 3-manifold. Without assuming the completeness of metrics, we prove the following

THEOREM 5.1. Let $(M, g)$ be a Lorentzian 3-manifold and $\left(D^{2} \times S^{1}, \mathcal{F}_{R}\right)$ a Reeb component of a codimension-1, totally geodesic foliation $\mathcal{F}$ of $M$. Then all inner leaves of $\mathcal{F}_{R}$ are spacelike, and the boundary leaf $\partial\left(D^{2} \times S^{1}\right)$ is lightlike.

The following corollary is an answer to Question 1.2 when the manifold is of dimension three.

COROLlary 5.9. Let $(M, g)$ be a closed Lorentzian 3-manifold with finite fundamental group and $\mathcal{F}$ a codimension-1, totally geodesic foliation of $M$. Then $\mathcal{F}$ consists of at least two kinds of leaves among spacelike, timelike, and lightlike ones.

Theorem 3.1 is a part of the author's doctoral thesis ([13]), which is not published elsewhere. Throughout this paper, we assume that manifolds, foliations and metrics under consideration are smooth.

2. Preliminaries. We recall several basic definitions and results about totally geodesic foliations of Lorentzian manifolds. Section 2.1 is devoted to basic definitions. Section 2.2 contains several results in [12] and a corollary. In Section 2.3 we recall the definition of Heegaard splittings of genus one, which will be used in Section 3.

\subsection{Definitions.}

Definition 2.1. Let $M$ be a smooth orientable manifold. A Lorentzian metric $g$ on $M$ is a nondegenerate, symmetric, covariant 2-tensor of signature $(+, \cdots,+,-)$. We call $(M, g)$ a Lorentzian manifold.

Definition 2.2. Let $g$ be a Lorentzian metric. A subspace $E \subset T_{x} M$ is called spacelike (resp. timelike, lightlike) if the signature of the induced metric $\left.g\right|_{E}$ is $(+, \cdots,+$ ) (resp. $(+, \cdots,+,-),(+, \cdots,+, 0))$. A vector $v \in T_{x} M$ is called spacelike (resp. timelike, lightlike) if $g(v, v)>0$ (resp. $g(v, v)<0, g(v, v)=0$ ).

For a Lorentzian manifold, it is well-known that there exists the Levi-Civita connection, that is, a connection which is torsion-free and compatible with the metric (see [8]). 
DEFINITION 2.3. A Lorentzian metric $g$ on a manifold $M$ is called time-orientable if there exists a non-singular, timelike vector field defined on entire $M$. When we fix a nonsingular, timelike vector field $T$ on $M$, a Lorentzian metric $g$ is called time-oriented, and a lightlike or timelike vector $v \in T_{x} M$ satisfying $g(v, T)<0$ is called positive (in a usual term, future-directed).

Positive lightlike or timelike vectors have the following good property (for a proof, see [8]).

Proposition 2.4 ([8]). Let $(M, g)$ and $T$ be as the above definition. Define

$$
\begin{aligned}
& C\left(\left.T\right|_{x}\right)=\left\{v \in T_{x} M \mid v \text { is timelike and } g\left(v,\left.T\right|_{x}\right)<0\right\}, \\
& \bar{C}\left(\left.T\right|_{x}\right)=\left\{v \in T_{x} M \mid v \text { is timelike or lightlike, and } g\left(v,\left.T\right|_{x}\right)<0\right\} .
\end{aligned}
$$

Then we have $a v+b w \in C\left(\left.T\right|_{x}\right)\left(\operatorname{resp} . \bar{C}\left(\left.T\right|_{x}\right)\right)$ for any $v, w \in C\left(\left.T\right|_{x}\right)\left(\operatorname{resp} . \bar{C}\left(\left.T\right|_{x}\right)\right)$, $a \geq 0$ and $b>0$.

DEFINITION 2.5. A Lorentzian metric $g$ is called (geodesically) complete if an affine parameter of each geodesic can be defined on the entire $\boldsymbol{R}$. Otherwise $g$ is called (geodesically) incomplete. A Lorentzian metric $g$ is called lightlike (geodesically) complete if an affine parameter of each geodesic with a lightlike initial vector can be defined on the entire $\boldsymbol{R}$.

REMARK 2.6. Even if a manifold is closed, a Lorentzian metric is not always complete.

DEFINITION 2.7. A foliation $\mathcal{F}$ of a Riemannian or Lorentzian manifold $(M, g)$ is called totally geodesic if each leaf $L$ of $\mathcal{F}$ is a totally geodesic submanifold, that is, a submanifold with the property that any geodesic with any initial vector in $T L$ is contained in $L$.

Definition 2.8. A foliation $\mathcal{F}$ of a manifold $M$ is called Lorentzian geodesible if there is a Lorentzian metric $g$ on $M$ for which $\mathcal{F}$ is totally geodesic. We call $\mathcal{F}$ complete Lorentzian geodesible if we can choose $g$ to be a complete Lorentzian metric. We define a lightlike complete Lorentzian geodesible foliation, in a natural fashion.

DEFINITION 2.9. Let $L$ be a submanifold in a Lorentzian manifold $(M, g)$. We call $L$ spacelike (resp. timelike, lightlike) if the tangent space $T_{x} L$ of $L$ at $x$ is a spacelike (resp. timelike, lightlike) subspace of $T_{x} M$ for each $x \in L$.

We can easily prove the following proposition.

PROPOSITION 2.10. Every leaf L of a totally geodesic foliation of a Lorentzian manifold is a spacelike, timelike, or lightlike submanifold.

Based on this proposition, we can call a leaf $L$ a spacelike leaf, a timelike leaf, or a lightlike leaf when $L$ is spacelike, timelike, or lightlike, respectively. 
We introduce the concept of "an element of isometric holonomy", which was called "an element of holonomy" in [12], and is a generalization of "an element of holonomy" stated in [1].

Definition 2.11. Let $\mathcal{H}$ be a distribution. A piecewise smooth curve $\sigma:\left[0, t_{0}\right] \rightarrow M$ is called an $\mathcal{H}$-curve if its tangent vectors lie in $\mathcal{H}$. An element of isometric holonomy along the $\mathcal{H}$-curve $\sigma$ is a family of maps $\left\{\psi_{t}: V_{\sigma(0)} \rightarrow V_{\sigma(t)}\right\}_{t \in\left[0, t_{0}\right]}$ which satisfies the following:

(1) The set $V_{\sigma(t)}$ is a plaque of the leaf containing the point $\sigma(t)$ for each $t \in\left[0, t_{0}\right]$. $\left[0, t_{0}\right]$

(2) The map $\psi_{t}$ is an isometry from $\left(V_{\sigma(0)},\left.g\right|_{V_{\sigma(0)}}\right)$ to $\left(V_{\sigma(t)},\left.g\right|_{V_{\sigma(t)}}\right)$ for each $t \in$

(3) The curve $\psi_{t}(x)$ with parameter $t \in\left[0, t_{0}\right]$ is an $\mathcal{H}$-curve for each $x \in V_{\sigma(0)}$ and $\psi_{t}(\sigma(0))=\sigma(t)$.

(4) The map $\psi_{0}$ is the identity map of $V_{\sigma(0)}$.

Definition 2.12. Let $\mathcal{F}$ be a foliation of $M$. A distribution $\mathcal{D}$ is called an Ehresmann connection for $\mathcal{F}$ if $\mathcal{D}$ satisfies the following:

(1) $T M=T \mathcal{F} \oplus \mathcal{D}$.

(2) For every $\mathcal{F}$-curve $\tau: I \rightarrow M$ and every $\mathcal{D}$-curve $\sigma: I \rightarrow M$ with the same initial point $\sigma(0)=\tau(0)$, there exists a map $\delta: I \times I \rightarrow M$ such that for every fixed $s$ the curve $\delta(\cdot, s)$ is a $\mathcal{D}$-curve and $\delta(\cdot, 0)=\sigma(\cdot)$, and for every fixed $t$ the curve $\delta(t, \cdot)$ is an $\mathcal{F}$-curve and $\delta(0, \cdot)=\tau(\cdot)$.

2.2. Relevant results about totally geodesic foliations. We state several relevant results about totally geodesic foliations. We first recall an equation discriminating whether a foliation is totally geodesic or not.

PROPOSITION 2.13 ([12]). Let $(M, g)$ be a pseudo-Riemannian manifold and $\mathcal{F}$ a codimension $k$ foliation of $M$. Then $\mathcal{F}$ is totally geodesic if and only if $\left(\mathcal{L}_{X} g\right)(Y, Z)=0$ for all $X \in \Gamma\left((T \mathcal{F})^{\perp}\right)$ and $Y, Z \in \Gamma(T \mathcal{F})$, where $(T \mathcal{F})^{\perp}$ is the distribution consisting of all vectors perpendicular to $T \mathcal{F}$.

Now we review the concept of the STL-decomposition.

Definition 2.14. Let $(M, g)$ be a Lorentzian manifold and $\mathcal{F}$ a codimension- $k$, totally geodesic foliation of $M$. Denote the union of all spacelike leaves, timelike ones, and lightlike ones of $\mathcal{F}$ by $\boldsymbol{S}, \boldsymbol{T}$ and $\boldsymbol{L}$, respectively. The decomposition $M=\boldsymbol{S} \sqcup \boldsymbol{T} \sqcup \boldsymbol{L}$ (disjoint union) is called the $S T L$-decomposition of $M$ by $\mathcal{F}$.

The STL-decomposition satisfies the following

Proposition 2.15 ([12]). The sets $\boldsymbol{S}$ and $\boldsymbol{T}$ are open in $M$, and $\boldsymbol{L}$ is closed in $M$.

Totally geodesic foliations of a lightlike complete Lorentzian 2-torus have the following property. 
THEOREM 2.16 ([12]). Let $\left(T^{2}, g\right)$ be a lightlike complete Lorentzian 2-torus. There exists no totally geodesic foliation containing at least two kinds of leaves among spacelike, timelike, and lightlike ones.

We have an easy corollary of this theorem.

COROllary 2.17. Let $(M, g)$ be a Lorentzian manifold. Assume that there is a timelike totally geodesic submanifold $N$ diffeomorphic to the 2-torus such that there is a totally geodesic foliation of $N$ consisting of at least two kinds of leaves among spacelike, timelike, and lightlike ones. Then $g$ is lightlike incomplete.

Finally, we review a result about an element of isometric holonomy. Let $\mathcal{F}$ be a totally geodesic foliation of a Lorentzian manifold $(M, g)$ and $\mathcal{H}$ the distribution perpendicular to $T \mathcal{F}$.

PROPOSITION 2.18 ([12]). If an $\mathcal{H}$-curve $\sigma:\left[0, t_{0}\right] \rightarrow M$ intersects only spacelike or timelike leaves, then there exists an element of isometric holonomy along $\sigma$.

2.3. Heegaard splittings of genus one. We recall Heegaard splittings of genus one of closed 3-manifolds. See [6] for further detail about Heegaard splittings.

Let $V_{1}$ and $V_{2}$ denote two copies of oriented $D^{2} \times S^{1} \subset \boldsymbol{C} \times \boldsymbol{C}$, where $D^{2}$ denotes a 2disk. Let $f: \partial V_{2} \rightarrow \partial V_{1}$ be an orientation reversing diffeomorphism. Consider a topological space $V_{1} \cup_{f} V_{2}$ and give it a differentiable structure in a certain way. We call the resulting manifold $M$. The couple $\left(V_{1}, V_{2}\right)$ is called a Heegaard splitting of genus one of $M$. Define curves $l, m$ by $l: t \mapsto\left(1, e^{2 \pi i t}\right) \in D^{2} \times S^{1}$ and $m: t \mapsto\left(e^{2 \pi i t}, 1\right)$. A simple closed curve in $\partial\left(D^{2} \times S^{1}\right)$ is called a meridian (resp. longitude) if it is homotopic to $m$ (resp. $l$ ) on $\partial\left(D^{2} \times S^{1}\right)$. The fundamental group $\pi_{1}\left(\partial\left(D^{2} \times S^{1}\right)\right)$ is isomorphic to $\langle l \mid-\rangle \oplus\langle m \mid-\rangle$, where $\langle l \mid-\rangle$ denotes the free group generated by $l$.

Let $\left(V_{1}, V_{2}\right)$ be a Heegaard splitting of $M$ and $f: \partial V_{2} \rightarrow \partial V_{1}$ a gluing map defining $M$. Let $l_{i}$ and $m_{i}$ be a longitude and a meridian in $V_{i}$, respectively. It is known that if $f_{*}\left(m_{2}\right)=$ $p l_{1}+q m_{1} \in \pi_{1}\left(\partial V_{1}\right)$, then $(p, q)=1$. When $f_{*}\left(m_{2}\right)=p l_{1}+q m_{1}$, we denote $M$ by $L(p, q)$. It is known that $L(0,1) \cong S^{2} \times S^{1}$ and $L(1,0) \cong S^{3}$. When $L(p, q)$ is diffeomorphic to neither $S^{2} \times S^{1}$ nor $S^{3}$, it is called a lens space of type $(p, q)$.

3. Constructions of Lorentzian geodesible foliations. The goal of this section is the following

THEOREM 3.1. If a closed 3-manifold has a Heegaard splitting of genus one, then it has a codimension-1 Lorentzian geodesible foliation.

3.1. Examples and propositions. First, we construct an example of a codimension-1, Lorentzian geodesible foliation of $S^{n-1} \times S^{1}$. 
EXAmple 3.2. Let $\tilde{M}=\boldsymbol{R}^{n} \backslash(0, \ldots, 0)$. Define the Lorentzian metric $\tilde{g}$ on $\tilde{M}$ by

$$
\tilde{g}=\frac{1}{\sum_{i=1}^{n} x_{i}^{2}}\left(\sum_{i=1}^{n-1} d x_{i} \otimes d x_{i}-d x_{n} \otimes d x_{n}\right)
$$

where $\left(x_{1}, \ldots, x_{n}\right)$ is the canonical coordinates of $\boldsymbol{R}^{n}$. Define the vector field $\tilde{X}$ by

$$
\tilde{X}=\sum_{i=1}^{n} x_{i} \frac{\partial}{\partial x_{i}}
$$

which is a Killing field on $\tilde{M}$. The distribution $T \tilde{\mathcal{F}}$ defined by $\operatorname{ker} \tilde{g}(\tilde{X}, \cdot)$ is completely integrable, and hence defines a totally geodesic foliation $\tilde{\mathcal{F}}$ by Proposition 2.13. The map $\psi:\left(x_{1}, \ldots, x_{n}\right) \mapsto\left(2 x_{1}, \ldots, 2 x_{n}\right)$ is an isometry preserving $\tilde{\mathcal{F}}$. Thus we have a manifold $M=\tilde{M} / \sim$, a Lorentzian metric $g$ on $M$, and a codimension-1, totally geodesic foliation $\mathcal{F}$ of $M$. Clearly, $M$ is diffeomorphic to $S^{n-1} \times S^{1}$. Hence $\mathcal{F}$ is a Lorentzian geodesible foliation of $S^{n-1} \times S^{1}$.

REMARK 3.3. The Lorentzian manifold $(M, g)$ obtained above is lightlike incomplete, because $\left\{x_{1}=\cdots=x_{n-2}=0\right\} / \sim$ satisfies the assumption of Corollary 2.17.

Second, we construct a totally geodesic foliation of $D^{2} \times S^{1}$.

EXAMPLE 3.4. Recall Example 3.2. Consider the case when $n=3$, that is,

$$
\left(\boldsymbol{R}^{3} \backslash\{0\}, \tilde{g}=\frac{1}{\sum_{i=1}^{3} x_{i}^{2}}\left(\sum_{i=1}^{2} d x_{i} \otimes d x_{i}-d x_{3} \otimes d x_{3}\right)\right) .
$$

Put

$$
M_{+}=\left\{\left(x_{1}, x_{2}, x_{3}\right) \mid x_{1}^{2}+x_{2}^{2}-x_{3}^{2} \leq 0, x_{3}>0\right\} .
$$

Define the diffeomorphism $\Phi: M_{+} \rightarrow D^{2}(1) \times \boldsymbol{R}$ by

$$
\left(x_{1}, x_{2}, x_{3}\right) \mapsto\left(\frac{\sqrt{2} x_{1}}{\sqrt{x_{1}^{2}+x_{2}^{2}+x_{3}^{2}}}, \frac{\sqrt{2} x_{2}}{\sqrt{x_{1}^{2}+x_{2}^{2}+x_{3}^{2}}}, \log \sqrt{x_{1}^{2}+x_{2}^{2}+x_{3}^{2}}\right) .
$$

The Lorentzian metric $\left(\Phi^{-1}\right)^{*} \tilde{g}$ on $D^{2}(1) \times \boldsymbol{R}$ is invariant by the additive $\boldsymbol{R}$-action. Hence we have the Lorentzian metric $g_{1}^{\prime}$ on $D^{2}(1) \times \boldsymbol{R} / 2 \pi \boldsymbol{Z}$.

Let $(x, y, t)$ denote coordinates of $D^{2}(1) \times S^{1}$, where $(x, y)$ and $(t)$ are the canonical coordinates of $\boldsymbol{R}^{2}$ and $\boldsymbol{R}$, respectively. We then have

$$
g_{1}^{\prime}=\left(\begin{array}{ccc}
1 / 2-x^{2} /\left(4-2 x^{2}-2 y^{2}\right) & -x y /\left(4-2 x^{2}-2 y^{2}\right) & x \\
-x y /\left(4-2 x^{2}-2 y^{2}\right) & 1 / 2-y^{2} /\left(4-2 x^{2}-2 y^{2}\right) & y \\
x & y & x^{2}+y^{2}-1
\end{array}\right),
$$

where the right hand side is the matrix of components of $g_{1}^{\prime}$ with respect to $(x, y, t)$. The foliation defined by $\operatorname{ker} g_{1}^{\prime}(\partial / \partial t, \cdot)$ is totally geodesic by Proposition 2.13 . 
Third, we change $g_{1}^{\prime}$ with a Lorentzian metric $g_{1}$ as follows. We change coordinates from $(x, y, t)$ to $(r, \theta, t)$, where $x=r \cos \theta$ and $y=r \sin \theta$. Then we have

$$
g_{1}^{\prime}=\left(\begin{array}{ccc}
\left(r^{2}-1\right) /\left(r^{2}-2\right) & 0 & r \\
0 & r^{2} / 2 & 0 \\
r & 0 & r^{2}-1
\end{array}\right)
$$

with respect to $(r, \theta, t)$. Consider a $C^{\infty}$ monotone increasing function $a:[0,1] \rightarrow[0,1]$ satisfying the following conditions:

(1) $a(0)=0$ and $a(1)=1$.

(2) There exists an $\varepsilon>0$ such that $a(r)=r$ for all $r \in[0, \varepsilon)$.

(3) $\left(d^{n} a / d r^{n}\right)(1)=0$ for all integer $n>0$.

We change $r$ with $a(r)$. The resulting metric is just $g_{1}$ in the next example.

EXAMPle 3.5. Consider $D^{2}(1) \times S^{1}$, where $D^{2}(1)$ is the unit 2-disk in $\boldsymbol{R}^{2}$ and $S^{1}=$ $\boldsymbol{R} / 2 \pi \boldsymbol{Z}$. We denote the coordinates of $D^{2}(1) \times S^{1}$ by $(x, y, t)$, where $(x, y)$ and $(t)$ are the canonical coordinates of $\boldsymbol{R}^{2}$ and $\boldsymbol{R}$, respectively. Define the Lorentzian metric $g_{1}$ by

$$
g_{1}=\left(\begin{array}{ccc}
\frac{G_{11}}{2\left(a^{2}-2\right)\left(x^{2}+y^{2}\right)^{2}} & \frac{G_{12}}{2\left(a^{2}-2\right)\left(x^{2}+y^{2}\right)^{2}} & \frac{a x}{\sqrt{x^{2}+y^{2}}} \\
\frac{G_{12}}{2\left(a^{2}-2\right)\left(x^{2}+y^{2}\right)^{2}} & \frac{G_{22}}{2\left(a^{2}-2\right)\left(x^{2}+y^{2}\right)^{2}} & \frac{a y}{\sqrt{x^{2}+y^{2}}} \\
\frac{a x}{\sqrt{x^{2}+y^{2}}} & \frac{a y}{\sqrt{x^{2}+y^{2}}} & a^{2}-1
\end{array}\right),
$$

where

$$
\begin{aligned}
& G_{11}=2\left(a^{2}-1\right) x^{4}+\left(a^{4}+2\left(x^{2}-1\right) a^{2}-2 x^{2}\right) y^{2}, \\
& G_{12}=x y\left(-a^{4}+2\left(x^{2}+y^{2}+1\right) a^{2}-2\left(x^{2}+y^{2}\right)\right), \\
& G_{22}=a^{2}\left(a^{2}-2\right) x^{2}+2\left(a^{2}-1\right) y^{2}\left(x^{2}+y^{2}\right) .
\end{aligned}
$$

Here, $a$ is the function $a\left(\sqrt{x^{2}+y^{2}}\right)$ defined above, the right hand side denotes the matrix of components of the metric with respect to coordinates $(x, y, t)$, and we assume that the numerator is divided by the denominator in $0 \leq \sqrt{x^{2}+y^{2}}<\varepsilon$ in each component of the matrix.

The vector field $\partial / \partial t$ is a Killing field, because all the components of the matrix are independent of $t$. Since

$$
g_{1}(\partial / \partial t, \cdot)=\frac{a x}{\sqrt{x^{2}+y^{2}}} d x+\frac{a y}{\sqrt{x^{2}+y^{2}}} d y+\left(a^{2}-1\right) d t,
$$

the foliation defined by $\operatorname{ker}\left(g_{1}(\partial / \partial t, \cdot)\right)$ is totally geodesic by Proposition 2.13. This foliation is a Reeb foliation. Clearly, $\partial\left(D^{2} \times S^{1}\right)$ is a lightlike leaf, and the other leaves are spacelike.

Fourth, we want to glue two copies of Example 3.5 together. Since it is not possible in general, we adopt the following tricks to accomplish it. 
Proposition 3.6. Let $\left(M_{i}, g_{i}\right)$ be a Lorentzian manifold with boundary, $N_{i}$ a connected component of $\partial M_{i}$, and $h_{i}: N_{i} \times[0,1] \rightarrow M_{i}$ a collar neighborhood such that $h_{i}\left(N_{i} \times 0\right)=N_{i}(i=1,2)$. Let $f: N_{1} \rightarrow N_{2}$ be a diffeomorphism. Denote $M_{1} \bigcup_{f} M_{2}$ by $M$. Define a differentiable structure on $M$ such that

$$
\begin{aligned}
h: N_{1} \times[-1,1] & \rightarrow M=M_{1} \cup_{f} M_{2} \\
(x, t) & \mapsto\left\{\begin{array}{l}
h_{1}(x, t) \text { for } t \geq 0 \\
h_{2}(f(x),-t) \text { for } t \leq 0
\end{array}\right.
\end{aligned}
$$

is a diffeomorphism into $M$ in a certain way. Assume that for all $x \in N_{1}$ there exists a local coordinate system $\left(x_{1}, \ldots, x_{n-1}\right)$ around $x$ such that the local coordinate system $\left(x_{1}, \ldots, x_{n-1}, t\right)$ in $h\left(N_{1} \times[-1,1]\right) \subset M$ satisfies the following:

(1) The $(i, j)$-component of $g_{1}$ equals the $(i, j)$-component of $g_{2}$ on $h\left(N_{1} \times 0\right)$ for all $i$ and $j$.

(2) There exists an $\varepsilon_{1}>0$ such that all the components of $g_{1}$ are functions depend on only $t$ on $h\left(N_{1} \times\left[0, \varepsilon_{1}\right)\right)$, and their differentials of order $\geq 1$ vanish on $h\left(N_{1} \times 0\right)$.

(3) There exists an $\varepsilon_{2}>0$ such that all the components of $g_{2}$ are functions depending only on $t$ on $h\left(N_{1} \times\left(-\varepsilon_{2}, 0\right]\right)$, and their differentials of order $\geq 1$ vanish on $h\left(N_{1} \times 0\right)$.

Then

$$
g= \begin{cases}g_{1} & \text { on } M_{1} \subset M \\ g_{2} & \text { on } M_{2} \subset M\end{cases}
$$

is a $C^{\infty}$ Lorentzian metric on $M$.

PROPOSITION 3.7. Let $V_{1}$ and $V_{2}$ be oriented manifolds with compact connected boundary. Let $f: \partial V_{2} \rightarrow \partial V_{1}$ be an orientation reversing diffeomorphism. Put $M=V_{1} \bigcup_{f} V_{2}$. Consider $\partial V_{1} \times[0,1]$, and regard $\mathrm{id}_{\partial V_{1}}$ and $f$ as

$$
\operatorname{id}_{\partial V_{1}}: \partial V_{1} \rightarrow \partial V_{1} \times 0 \text { and } f: \partial V_{2} \rightarrow \partial V_{1} \times 1
$$

If we take the orientation of $V_{1} \times[0,1]$ such that $f: \partial V_{2} \rightarrow \partial V_{1} \times 1$ is orientation reversing, then the map id $: \partial V_{1} \rightarrow \partial V_{1} \times 0$ is orientation reversing, and $M$ is diffeomorphic to $V_{1} \bigcup_{\text {id }}\left(\partial V_{1} \times[0,1]\right) \bigcup_{f} V_{2}$.

3.2. The proof of Theorem 3.1. We will construct a totally geodesic foliation of $L(p, q)$. Let $V_{1}$ and $V_{2}$ be two copies of an oriented $D^{2}(1) \times S^{1}$. Consider $\left(V_{i}, g_{1}\right)$, where $g_{1}$ is the Lorentzian metric defined in Example 3.5. We change coordinates from $\left(x_{i}, y_{i}, t_{i}\right) \in V_{i}$ to $\left(r_{i}, \theta_{i}, t_{i}\right)$, where $x_{i}=r_{i} \cos \theta_{i}$ and $y_{i}=r_{i} \sin \theta_{i}$. We regard the $r_{i}$-direction as a "collar direction," that is, the collar neighborhood is defined by

$$
h_{i}: \partial V_{i} \times[0, \varepsilon] \rightarrow V_{i}, \quad\left(\theta_{i}, t_{i}, s_{i}\right) \mapsto\left(1-s_{i}, \theta_{i}, t_{i}\right) .
$$

Consider the gluing map $f: \partial V_{2} \cong \boldsymbol{R}^{2} / 2 \pi \boldsymbol{Z}^{2} \rightarrow \partial V_{1} \cong \boldsymbol{R}^{2} / 2 \pi \boldsymbol{Z}^{2}$ defined by

$$
f:\left(\begin{array}{c}
\theta_{2} \\
t_{2}
\end{array}\right) \mapsto\left(\begin{array}{cc}
q & r^{\prime} \\
p & s^{\prime}
\end{array}\right)\left(\begin{array}{c}
\theta_{2} \\
t_{2}
\end{array}\right) \quad \text { for some } r^{\prime}, s^{\prime} \in \boldsymbol{Z},
$$


where $q s^{\prime}-p r^{\prime}=-1$. Note that $f$ determines $L(p, q)$. In general, we cannot glue $\left(V_{1}, g_{1}\right)$ and $\left(V_{2}, g_{1}\right)$ by using only Proposition 3.6. So we use Proposition 3.7 to get a Lorentzian metric and a totally geodesic foliation of $L(p, q)$. We will carry out this by several steps.

Step 1. Denote coordinates of $\partial V_{1} \times[0,1]$ by $(\theta, t, s)$, where $\theta=\theta_{1}$ and $t=t_{1}$. Consider the glued manifold $V_{1} \bigcup_{\text {id }}\left(\partial V_{1} \times[0,1]\right) \bigcup_{f} V_{2}$.

LEMMA 3.8. We can join the metric restricted on $\partial V_{1} \times 0$ to the metric on $\partial V_{1} \times 1$ so that the constructed metric $g^{\prime}$ on $\partial V_{1} \times[0,1]$ satisfies the following conditions:

(1) All the components of $g^{\prime}$ with respect to $(\theta, t, s)$ depend only on $s \in[0,1]$.

(2) The manifold $\partial V_{1} \times\{s\}$ is lightlike for all $s \in[0,1]$.

Proof. Note that

$$
g_{1}=\left(\begin{array}{ccc}
\left(a^{2}-1\right) /\left(a^{2}-2\right) & 0 & a \\
0 & a^{2} / 2 & 0 \\
a & 0 & a^{2}-1
\end{array}\right)
$$

where the right hand side is the matrix of components of $g_{1}$ with respect to $\left(r_{i}, \theta_{i}, t_{i}\right) \in V_{i}$. Hence we have

$$
g_{1}=\left(\begin{array}{ccc}
a^{2} / 2 & 0 & 0 \\
0 & a^{2}-1 & -a \\
0 & -a & \left(a^{2}-1\right) /\left(a^{2}-2\right)
\end{array}\right)
$$

with respect to the collar coordinates $\left(\theta_{i}, t_{i}, s_{i}\right) \in \partial V_{i} \times[0, \varepsilon]$. Since

$$
g_{1}=\left(\begin{array}{ccc}
1 / 2 & 0 & 0 \\
0 & 0 & -1 \\
0 & -1 & 0
\end{array}\right)
$$

on $\partial V_{i} \times 0 \subset \partial V_{i} \times[0, \varepsilon]$, the metric on $\partial V_{1} \times 0 \subset \partial V_{1} \times[0,1]$ is represented by

$$
\left(\begin{array}{ccc}
1 & 0 & 0 \\
0 & 1 & 0 \\
0 & 0 & -1
\end{array}\right)\left(\begin{array}{ccc}
1 / 2 & 0 & 0 \\
0 & 0 & -1 \\
0 & -1 & 0
\end{array}\right)\left(\begin{array}{ccc}
1 & 0 & 0 \\
0 & 1 & 0 \\
0 & 0 & -1
\end{array}\right)=\left(\begin{array}{ccc}
1 / 2 & 0 & 0 \\
0 & 0 & 1 \\
0 & 1 & 0
\end{array}\right)
$$

with respect to the coordinates $(\theta, t, s) \in \partial V_{1} \times[0,1]$. Note that the inverse map $f^{-1}$ : $\partial V_{1} \times 1 \rightarrow \partial V_{2}$ is represented by

$$
\left(\begin{array}{cc}
-s^{\prime} & r^{\prime} \\
p & -q
\end{array}\right)
$$

Hence we have

$$
\left(\begin{array}{ccc}
-s^{\prime} & p & 0 \\
r^{\prime} & -q & 0 \\
0 & 0 & 1
\end{array}\right)\left(\begin{array}{ccc}
1 / 2 & 0 & 0 \\
0 & 0 & -1 \\
0 & -1 & 0
\end{array}\right)\left(\begin{array}{ccc}
-s^{\prime} & r^{\prime} & 0 \\
p & -q & 0 \\
0 & 0 & 1
\end{array}\right)=\left(\begin{array}{ccc}
s^{2} / 2 & -r^{\prime} s^{\prime} / 2 & -p \\
-r^{\prime} s^{\prime} / 2 & r^{\prime 2} / 2 & q \\
-p & q & 0
\end{array}\right)
$$

on $\partial V_{1} \times 1 \subset \partial V_{1} \times[0,1]$ with respect to $(\theta, t, s) \in \partial V_{1} \times[0,1]$. (See Figure 1.)

Consider a $C^{\infty} G L(2, \boldsymbol{R})$-valued function

$$
\left(\begin{array}{ll}
b_{s} & c_{s} \\
d_{s} & k_{s}
\end{array}\right), \quad s \in[0,1]
$$




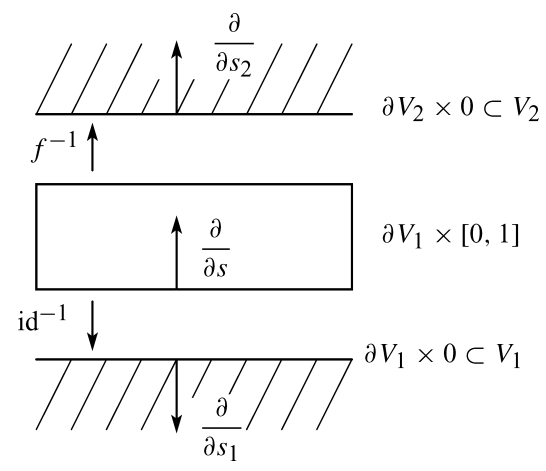

FIGURE 1. $f$ and id.

satisfying

$$
\left(\begin{array}{cc}
b_{0} & c_{0} \\
d_{0} & k_{0}
\end{array}\right)=\left(\begin{array}{cc}
1 & 0 \\
0 & -1
\end{array}\right) \in\left(\begin{array}{cc}
1 & 0 \\
0 & -1
\end{array}\right) G L_{+}(2, \boldsymbol{R})
$$

and

$$
\left(\begin{array}{ll}
b_{1} & c_{1} \\
d_{1} & k_{1}
\end{array}\right)=\left(\begin{array}{cc}
-s^{\prime} & r^{\prime} \\
p & -q
\end{array}\right) \in\left(\begin{array}{cc}
1 & 0 \\
0 & -1
\end{array}\right) G L_{+}(2, \boldsymbol{R}) .
$$

Note that such a function exists. Put

$$
\begin{aligned}
g^{\prime} & =\left(\begin{array}{ccc}
b_{s} & d_{s} & 0 \\
c_{s} & k_{s} & 0 \\
0 & 0 & 1
\end{array}\right)\left(\begin{array}{ccc}
1 / 2 & 0 & 0 \\
0 & 0 & -1 \\
0 & -1 & 0
\end{array}\right)\left(\begin{array}{ccc}
b_{s} & c_{s} & 0 \\
d_{s} & k_{s} & 0 \\
0 & 0 & 1
\end{array}\right) \\
& =\left(\begin{array}{ccc}
b_{s}^{2} / 2 & b_{s} c_{s} / 2 & -d_{s} \\
b_{s} c_{s} / 2 & c_{s}^{2} / 2 & -k_{s} \\
-d_{s} & -k_{s} & 0
\end{array}\right) .
\end{aligned}
$$

Note that signature of this matrix is $(+,+,-)$. Thus we regard $g^{\prime}$ as the matrix of components of the Lorentzian metric with respect to $(\theta, t, s) \in \partial V_{1} \times[0,1]$.

Now we show that $g^{\prime}$ satisfies the condition (2). We have

$$
\left.g^{\prime}\right|_{\partial V_{1} \times\{s\}}=\left(\begin{array}{cc}
b_{s}^{2} / 2 & b_{s} c_{s} / 2 \\
b_{s} c_{s} / 2 & c_{s}^{2} / 2
\end{array}\right)
$$

with respect to $(\theta, t) \in \partial V_{1} \times\{s\}$. Thus $\partial V_{1} \times\{s\}$ is lightlike. This proves Lemma 3.8.

REMARK 3.9. The foliation $\left\{\partial V_{1} \times\{s\}\right\}_{s \in[0,1]}$ is a totally geodesic foliation of $\left(\partial V_{1} \times\right.$ $\left.[0,1], g^{\prime}\right)$ by (1), (2) and Proposition 2.13.

Step 2. We change the parameter $s$ of each component of $g^{\prime}$ to $u(s)$, where $u$ is a function satisfying the following:

(1) The function $u:[0,1] \rightarrow[0,1]$ is a $C^{\infty}$ monotone increasing function.

(2) $\left(d^{n} u / d s^{n}\right)(0)=\left(d^{n} u / d s^{n}\right)(1)=0$ for all integer $n>0$.

We denote a new metric by the same symbol $g^{\prime}$. 
Step 3. Put

$$
g=\left\{\begin{array}{lll}
g_{1} & \text { on } & V_{1}, \\
g^{\prime} & \text { on } & \partial V_{1} \times[0,1], \\
g_{1} & \text { on } & V_{2} .
\end{array}\right.
$$

Note that $g$ is a $C^{\infty}$ Lorentzian metric on $V_{1} \bigcup_{\text {id }}\left(\partial V_{1} \times[0,1]\right) \bigcup_{f} V_{2}$ by Proposition 3.6. We define the desired foliation $\mathcal{F}$ by

$$
\mathcal{F}=\left\{\begin{array}{lll}
\operatorname{ker}\left(g_{1}\left(\partial / \partial t_{1}, \cdot\right)\right) & \text { on } & V_{1}, \\
\left\{\partial V_{1} \times\{s\}\right\}_{s \in[0,1]} & \text { on } & \partial V_{1} \times[0,1], \\
\operatorname{ker}\left(g_{1}\left(\partial / \partial t_{2}, \cdot\right)\right) & \text { on } & V_{2} .
\end{array}\right.
$$

Note that $\mathcal{F}$ is a $C^{\infty}$ totally geodesic foliation. Hence we have a Lorentzian geodesible foliation of $L(p, q)$. This completes the proof.

4. Some properties of totally geodesic foliations. In this section, we study totally geodesic foliations consisting of spacelike leaves, and codimension-1, totally geodesic foliations consisting of timelike leaves of Lorentzian 3-manifolds. We do not assume the completeness of Lorentzian metrics.

First, we consider totally geodesic foliations consisting of spacelike leaves.

PROPOSITION 4.1. Let $(M, g)$ be a Lorentzian manifold with or without boundary and $\mathcal{F}$ a codimension- $k$, totally geodesic foliation consisting of spacelike leaves. Assume that $\mathcal{F}$ is tangent to $\partial M$ and the distribution $(T \mathcal{F})^{\perp}$ is integrable. Then for an arbitrary metric $h$ on $(T \mathcal{F})^{\perp}$, the foliation $\mathcal{F}$ is totally geodesic with respect to the Riemannian metric $\left.g\right|_{T} \mathcal{F}+h$.

Proof. By the assumption, we have $T M=T \mathcal{F} \oplus(T \mathcal{F})^{\perp}$. Put $g_{R}=\left.g\right|_{T \mathcal{F}}+h$. Then the distribution $T \mathcal{F}$ is perpendicular to $(T \mathcal{F})^{\perp}$ with respect to $g_{R}$. Let $\mathcal{H}$ be the foliation determined by $(T \mathcal{F})^{\perp}$. Since $\mathcal{F}$ is totally geodesic, we have

$$
\left(\mathcal{L}_{X} g\right)(Y, Z)=0 \text { for all } X \in \Gamma(T \mathcal{H}) \text { and } Y, Z \in \Gamma(T \mathcal{F}) \text {. }
$$

There exists a product neighborhood $U \times V$ for each $x \in M$, where $U$ is a plaque of $\mathcal{F}$ and $V$ is a plaque of $\mathcal{H}$. Hence there exist linear independent, $\mathcal{F}$-preserving, non-singular local vector fields $X_{1}, \ldots, X_{k} \in \Gamma(T \mathcal{H})$. If we describe $X \in \Gamma(T \mathcal{H})$ as $X=\sum a_{i} X_{i}$, then we have

$$
\begin{aligned}
\left(\mathcal{L}_{X} g_{R}\right)(Y, Z) & =\left(\mathcal{L}_{\left(\sum a_{i} X_{i}\right)} g_{R}\right)(Y, Z) \\
& =\sum a_{i}\left\{X_{i}\left(g_{R}(Y, Z)\right)-g_{R}\left(\left[X_{i}, Y\right], Z\right)-g_{R}\left(Y,\left[X_{i}, Z\right]\right)\right\} \\
& =\sum a_{i}\left\{X_{i}\left(\left(\left.g\right|_{T \mathcal{F}}\right)(Y, Z)\right)-\left(\left.g\right|_{T \mathcal{F}}\right)\left(\left[X_{i}, Y\right], Z\right)-\left(\left.g\right|_{T \mathcal{F}}\right)\left(Y,\left[X_{i}, Z\right]\right)\right\} \\
& =\sum a_{i}\left\{X_{i}(g(Y, Z))-g\left(\left[X_{i}, Y\right], Z\right)-g\left(Y,\left[X_{i}, Z\right]\right)\right\} \\
& =\sum a_{i}\left(\mathcal{L}_{X_{i}} g\right)(Y, Z)=0 .
\end{aligned}
$$

Therefore $\mathcal{F}$ is totally geodesic with respect to $g_{R}$.

If $M$ is closed, by Proposition 4.1 and [1] we can prove the following 
Proposition 4.2. Let $(M, g)$ be a compact Lorentzian manifold and $\mathcal{F}$ be a totally geodesic foliation consisting of spacelike leaves. Assume that $\mathcal{F}$ is tangent to $\partial M$ and $(T \mathcal{F})^{\perp}$ is completely integrable. Then the distribution $(T \mathcal{F})^{\perp}$ is an Ehresmann connection for $\mathcal{F}$.

PROOF. Let $\mathcal{H}$ be the foliation determined by $(T \mathcal{F})^{\perp}$. Cover $M$ by a product foliation charts $\left\{U_{i} \times V_{i}\right\}_{i=1}^{m}$, where $U_{i}$ is an $\mathcal{F}$-plaque and $V_{i}$ is an $\mathcal{H}$-plaque. Let $h$ be a metric on $(T \mathcal{F})^{\perp}$, and define the Riemannian metric $g_{R}$ by $\left.g\right|_{T \mathcal{F}}+h$. We have $T \mathcal{F} \perp(T \mathcal{F})^{\perp}$ with respect to $g_{R}$. Let $d$ be the distance determined by $g_{R}$. Consider a Lebesgue number $\rho$ with respect to $\left\{U_{i} \times V_{i}\right\}_{i=1}^{m}$, that is, any set with the diameter $<\rho$ is contained in $U_{i} \times V_{i}$ for some $i$. Hence any $\mathcal{F}$-plaque $P$ with the diameter $<\rho$ with respect to $\left.g_{R}\right|_{P}$ is contained in $U_{i} \times V_{i}$ for some $i$.

Let $\sigma:[0,1] \rightarrow M$ be an arbitrary $\mathcal{H}$-curve and $\tau:[0,1] \rightarrow M$ be an $\mathcal{F}$-curve with the length $<\rho / 2$. We will construct a map $\delta:[0,1] \times[0,1] \rightarrow M$ satisfying Definition $2.12(2)$. Decompose $[0,1]=\left[t_{0}, t_{1}\right] \cup\left[t_{1}, t_{2}\right] \cup \cdots \cup\left[t_{l-1}, t_{l}\right]$ so that $t_{0}=0, t_{l}=1$, and $\sigma\left(\left[t_{i-1}, t_{i}\right]\right)$ has the length $<\rho / 2$ for all $i$. The curve $\sigma\left(\left[t_{0}, t_{1}\right]\right) \cup \tau([0,1])$ has the length $<\rho$. Hence there exists an $i_{0}$ such that $\sigma\left(\left[t_{0}, t_{1}\right]\right) \cup \tau([0,1]) \subset U_{i_{0}} \times V_{i_{0}}$.

LEMMA 4.3. We can construct an element of isometric holonomy along $\left.\sigma\right|_{\left[t_{0}, t_{1}\right]}$

$$
\left\{\psi_{t}: V_{\sigma(0)} \rightarrow V_{\sigma(t)}\right\}_{t \in\left[t_{0}, t_{1}\right]}
$$

so that $V_{\sigma(0)}=U_{i_{0}} \times \sigma(0)$ and $V_{\sigma(t)}=U_{i_{0}} \times \sigma(t)$.

Proof. Let $p: U_{i_{0}} \times V_{i_{0}} \rightarrow V_{i_{0}}$ be the projection. Define the vector field $Y$ on $p \circ \sigma\left(\left[t_{0}, t_{1}\right]\right)$ tangent to $V_{i_{0}}$ by

$$
Y_{p \circ \sigma(t)}=\left.\frac{\mathrm{d}}{\mathrm{d} t}(p \circ \sigma)\right|_{t} .
$$

Define the vector field $X$ on $U_{i_{0}} \times \sigma\left(\left[t_{0}, t_{1}\right]\right)$ tangent to $\mathcal{H}$ by $p_{*} X=Y$. The vector field $X$ is $\mathcal{F}$-preserving and tangent to $\mathcal{H}$. Hence a local one-parameter group generated by $X$ determines an element of isometric holonomy along $\left.\sigma\right|_{\left[t_{0}, t_{1}\right]}$

$$
\left\{\psi_{t}: U_{i_{0}} \times \sigma(0) \rightarrow U_{i_{0}} \times \sigma(t)\right\}_{t \in\left[t_{0}, t_{1}\right]} .
$$

Define the map $\delta_{1}:\left[t_{0}, t_{1}\right] \times[0,1] \rightarrow M$ by

$$
\delta_{1}(t, s)=\psi_{t}(\tau(s)) .
$$

Put $\tau_{1}(s)=\delta_{1}\left(t_{1}, s\right)$. By the definition of elements of isometric holonomy, the curve $\tau_{1}$ has the length $<\rho / 2$. Construct $\delta_{2}:\left[t_{1}, t_{2}\right] \times[0,1] \rightarrow M$ by applying the same argument as above to $\sigma\left(\left[t_{1}, t_{2}\right]\right) \cup \tau_{1}([0,1])$. Repeat this process and define $\delta:[0,1] \times[0,1] \rightarrow M$ by

$$
\delta(t, s)=\delta_{i}(t, s) \quad \text { when } t \in\left[t_{i-1}, t_{i}\right] .
$$

This $\delta$ satisfies Definition $2.12(2)$. Therefore $(T \mathcal{F})^{\perp}$ is an Ehresmann connection for $\mathcal{F}$. This proves the proposition.

Now we consider codimension-1, totally geodesic foliations consisting of timelike leaves of Lorentzian 3-manifolds. 
Proposition 4.4. Let $M$ be an orientable 3-manifold, g a time-orientable Lorentzian metric on $M$, and $\mathcal{F}$ an orientable, codimension-1, totally geodesic foliation consisting of timelike leaves. Denote the foliation determined by $(T \mathcal{F})^{\perp}$ by $\mathcal{H}$. Then the following hold.

(1) The lightlike vectors on $T \mathcal{F}$ determine two subfoliations $\mathcal{L}_{0}, \mathcal{L}_{1}$ of $\mathcal{F}$.

(2) The distribution $T \mathcal{H} \oplus T \mathcal{L}_{i}$ is completely integrable for $i=0,1$. Therefore, if $\mathcal{H}_{i}$ denotes the foliation determined by $T \mathcal{H} \oplus T \mathcal{L}_{i}, i=0,1$, then $\left(\mathcal{F}, \mathcal{H}_{0}, \mathcal{H}_{1}\right)$ is a total foliation of $M$ (We call it the total foliation associated with $\mathcal{F}$ ).

Proof. Fix orientations of $M$ and $\mathcal{F}$. By the assumption of $g$, there exists a nonsingular, timelike vector field $T$ on $M$. Since all the leaves of $\mathcal{F}$ are timelike, we have a splitting $T M=T \mathcal{F} \oplus T \mathcal{H}$. Let $\pi: T M \rightarrow T \mathcal{F}$ denote the projection defined by the splitting $T M=T \mathcal{F} \oplus T \mathcal{H}$. By straight computation, $\pi(T)$ is non-singular and timelike. Hence for all $L \in \mathcal{F}$ we can regard $\left(L,\left.g\right|_{L}\right)$ as an oriented, time-oriented, Lorentzian 2-manifold by $\left.\pi(T)\right|_{L}$. Fix a leaf $L$ of $\mathcal{F}$ and a point $x \in L$. Define two lightlike subspaces $T_{x} \mathcal{L}_{0}, T_{x} \mathcal{L}_{1}$ of $T_{x} L$ by the following: Take linear independent, positive, lightlike vectors $V_{0}, V_{1}$ on $T_{x} L$ so that $\left\{V_{0}, V_{1}\right\}$ equals the orientation of $T_{x} L$, and put $T_{x} \mathcal{L}_{0}=\operatorname{Span}\left\{V_{0}\right\}$ and $T_{x} \mathcal{L}_{1}=\operatorname{Span}\left\{V_{1}\right\}$. Define distributions $T \mathcal{L}_{0}, T \mathcal{L}_{1}$ by

$$
T \mathcal{L}_{0}=\bigcup_{x \in M} T_{x} \mathcal{L}_{0}, \quad T \mathcal{L}_{1}=\bigcup_{x \in M} T_{x} \mathcal{L}_{1}
$$

which are $C^{\infty}$ subdistributions of $T \mathcal{F}$, proving (1).

We will prove that $T \mathcal{H} \oplus T \mathcal{L}_{i}$ is completely integrable for $i=0,1$. Let $\sigma:[0,1] \rightarrow M$ be an arbitrary $\mathcal{H}$-curve. Let $\left\{\psi_{t}: V_{\sigma(0)} \rightarrow V_{\sigma(t)}\right\}_{t \in[0,1]}$ be an element of isometric holonomy along $\sigma$. Since $\psi_{t}$ is an isometry, the map $\psi_{t}$ preserves $\mathcal{L}_{0}$ and $\mathcal{L}_{1}$. Define the vector field $H$ defined on $\bigcup_{t} V_{\sigma(t)}$ by

$$
H_{\psi_{t}(p)}=\frac{\mathrm{d}}{\mathrm{d} t} \psi_{t}(p) \text {. }
$$

Let $L_{i} \in \Gamma\left(\left.T \mathcal{L}_{i}\right|_{\cup_{t} V_{\sigma(t)}}\right)$ be a frame. We have $\left[H, L_{i}\right]=l_{i} L_{i}$ for some function $l_{i}$. Therefore $T \mathcal{H} \oplus T \mathcal{L}_{i}$ is integrable. This proves the proposition.

5. Reeb components of totally geodesic foliations of Lorentzian 3-manifolds. In this section, we study Reeb components of codimension-1, totally geodesic foliations of Lorentzian 3-manifolds. We do not assume the completeness of metrics under consideration. We have the following

THEOREM 5.1. Let $(M, g)$ be a Lorentzian 3-manifold and $\left(D^{2} \times S^{1}, \mathcal{F}_{R}\right)$ a Reeb component of a codimension-1, totally geodesic foliation $\mathcal{F}$ of $M$. Then all inner leaves of $\mathcal{F}_{R}$ are spacelike, and the boundary leaf $\partial\left(D^{2} \times S^{1}\right)$ is lightlike.

PROOF. By taking a finite covering of $M$, we can assume that $M$ is orientable, $g$ is time-orientable, and $\mathcal{F}$ is orientable. By the property of the STL-decomposition of $M$ by $\mathcal{F}$ (Proposition 2.15), we have the following three cases:

(i) The set $\boldsymbol{L} \cap\left(D^{2} \times S^{1}\right)$ is empty.

(ii) The set $\boldsymbol{L} \cap\left(D^{2} \times S^{1}\right)$ contains $\partial\left(D^{2} \times S^{1}\right)$ and does not equal $\partial\left(D^{2} \times S^{1}\right)$. 
(iii) The set $\boldsymbol{L} \cap\left(D^{2} \times S^{1}\right)$ equals $\partial\left(D^{2} \times S^{1}\right)$. Consequently, we have the following five cases:

(1) All the leaves of $\mathcal{F}_{R}$ are spacelike.

(2) All the leaves of $\mathcal{F}_{R}$ are timelike.

(3) The boundary leaf $\partial\left(D^{2} \times S^{1}\right)$ and at least one of the inner leaves are lightlike.

(4) All the inner leaves of $\mathcal{F}_{R}$ are timelike and the boundary is lightlike.

(5) All the inner leaves of $\mathcal{F}_{R}$ are spacelike and the boundary is lightlike.

We will prove that the case (1) through the case (4) do not occur in the following propositions. Therefore only the case (5) occurs.

PROPOSITION 5.2. The case (1) does not occur, that is, there exists no Reeb component $\left(D^{2} \times S^{1}, \mathcal{F}_{R}\right)$ of a codimension-1, totally geodesic foliation such that all the leaves of $\mathcal{F}_{R}$ are spacelike.

Proof. Assume that there is a Reeb component $\left(D^{2} \times S^{1}, \mathcal{F}_{R}\right)$ consisting of spacelike leaves. By Proposition 4.2, the distribution $\left(T \mathcal{F}_{R}\right)^{\perp}$ is an Ehresmann connection for $\mathcal{F}_{R}$. But an inner leaf never cover the boundary leaf for any normal distribution of $\mathcal{F}_{R}$, which is contradiction.

PROPOSITION 5.3. The case (2) does not occur, that is, there exists no Reeb component $\left(D^{2} \times S^{1}, \mathcal{F}_{R}\right)$ such that all the leaves of $\mathcal{F}_{R}$ are timelike.

We need two lemmas to prove Proposition 5.3.

LEMMA 5.4. Let $\left(M_{i}, g_{i}\right)$ be a pseudo-Riemannian manifold, $i=0,1$, and $\varphi:\left(M_{0}, g_{0}\right)$ $\rightarrow\left(M_{1}, g_{1}\right)$ be an isometry. Then the following hold:

(a) $\mathrm{d} \varphi\left(\nabla_{X} Y\right)=\nabla_{\mathrm{d} \varphi(X)} \mathrm{d} \varphi(Y)$ for all $X, Y \in \Gamma\left(T M_{0}\right)$, where $\nabla$ in the left (resp. right) hand side is the Levi-Civita connection of $g_{0}$ (resp. $g_{1}$ ).

(b) If $\exp _{x} X$ is defined, then $\varphi\left(\exp _{x} X\right)=\exp _{\varphi(x)} \mathrm{d} \varphi(X)$.

Proof. (a) Define $\hat{\nabla}_{X} Y=\mathrm{d} \varphi^{-1}\left(\nabla_{\mathrm{d} \varphi(X)} \mathrm{d} \varphi(Y)\right)$ for $X, Y \in \Gamma\left(T M_{0}\right)$. By a straight computation, we see that $\hat{\nabla}$ is the Levi-Civita connection. Hence $\hat{\nabla}=\nabla$.

(b) We can easily prove this by (a).

LEMMA 5.5. The identity component of $S O(1,1)$ is

$$
\left\{\left(\begin{array}{cc}
\sqrt{c^{2}+1} & c \\
c & \sqrt{c^{2}+1}
\end{array}\right) \mid c \in \boldsymbol{R}\right\}
$$

the eigenvalues of $\left(\begin{array}{cc}\sqrt{c^{2}+1} & c \\ c & \sqrt{c^{2}+1}\end{array}\right)$ are $\sqrt{c^{2}+1}+c$ and $\sqrt{c^{2}+1}-c$, and the eigenvectors are ${ }^{t}(1,1)$ and ${ }^{t}(1,-1)$ (these are lightlike). Moreover,

$$
\begin{aligned}
& \sqrt{c^{2}+1}+c>1>\sqrt{c^{2}+1}-c(c>0), \\
& \sqrt{c^{2}+1}-c>1>\sqrt{c^{2}+1}+c(c<0) .
\end{aligned}
$$




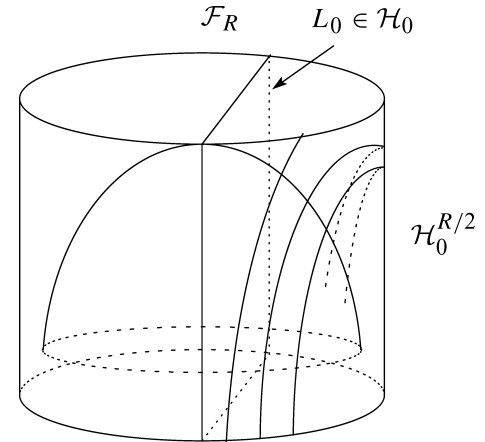

FIGURE 2. A half Reeb component in $\mathcal{H}_{0}$.

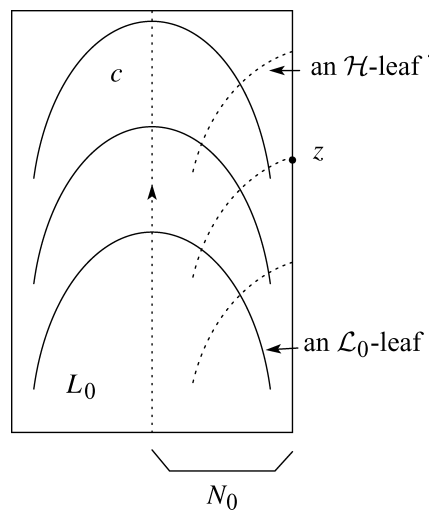

FIGURE $3 . \quad \mathcal{L}_{0}$ and $\mathcal{H}$ on $L_{0}$.

Proof of Proposition 5.3. Let $\left(\mathcal{F}_{R}, \mathcal{H}_{0}, \mathcal{H}_{1}\right)$ be the total foliation associated with $\mathcal{F}_{R}$. We use the same notation as in Proposition 4.4. By [11], there exists a half Reeb component $\mathcal{H}_{0}^{R / 2}$ in $\mathcal{H}_{0}$ and an annular leaf $L_{0} \in \mathcal{H}_{0}^{R / 2}$ (see Figure 2). The foliation $\mathcal{F}_{R} \cap L_{0}=\left.\mathcal{L}_{0}\right|_{L_{0}}$ is a Reeb foliation. Since $T \mathcal{H}_{0}=T \mathcal{H} \oplus T \mathcal{L}_{0}$, there is the foliation $\left.\mathcal{H}\right|_{L_{0}}$ on $L_{0}$. Take an arbitrary point $z \in \partial L_{0}$. Start from $z$ along $\mathcal{H}$ and consider the limit $\mathcal{H}$-leaf $c$. The leaf $c$ is closed. Put

$$
N_{0}=c \bigcup \text { (the connected component of } L_{0} \backslash c \text { containing } z \text { ). }
$$

All the $\mathcal{H}$-leaves in $N_{0} \backslash c$ approach $c$ (see Figure 3). Fix an arbitrary point $x \in c$. By parametrizing $c$, we define an $\mathcal{H}$-curve

$$
\sigma:[0,1] \rightarrow D^{2} \times S^{1}, \sigma(0)=\sigma(1)=x,
$$

assuming that $\sigma$ proceeds in the direction where all the $\mathcal{H}$-leaves in $N_{0} \backslash c$ leave from $c$. Let $L_{1} \in \mathcal{H}_{1}$ be the leaf passing through $c$. Consider a tubular neighborhood $T$ of $c$ in $L_{1}$, and let $N_{1}$ be the subset of $T$ which intersects $\mathcal{H}_{0}^{R / 2}$. All the $\mathcal{H}$-leaves near $c$ in $N_{1}$ leave from $c$ along $\sigma$ (see Figure 4).

Consider an element of isometric holonomy along $\sigma$

$$
\left\{\psi_{t}: V_{\sigma(0)} \rightarrow V_{\sigma(t)}\right\}_{t \in[0,1]},
$$

where $V_{\sigma(t)}$ is an $\mathcal{F}_{R}$-plaque. Let $i$ denote 0 or 1 . Note that the set $N_{i} \cap V_{\sigma(0)}$ is a subset of an $\mathcal{L}_{i}$-leaf. Fix a point $y^{(i)} \in N_{i} \cap V_{\sigma(0)} \backslash\{x\}$. Let $\alpha^{(i)}$ be the geodesic satisfying $\alpha^{(i)}(0)=x$ and $\alpha^{(i)}(1)=y^{(i)}$. The vector $\dot{\alpha}^{(i)}(0)$ is lightlike and $y^{(i)}=\exp _{x} \dot{\alpha}^{(i)}(0)$. The map $\psi_{1}$ is an isometry from $\left(V_{\sigma(0)},\left.g\right|_{V_{\sigma(0)}}\right)$ to $\left(V_{\sigma(1)},\left.g\right|_{V_{\sigma(1)}}\right)$. By Lemma 5.4 and $\psi_{1}(x)=x$, we have

$$
\psi_{1}\left(y^{(i)}\right)=\exp _{x} \mathrm{~d} \psi_{1}\left(\dot{\alpha}^{(i)}(0)\right) .
$$

All the $\mathcal{H}$-leaves near $c$ in $N_{i}$ leave from $c$ along $\sigma$. Hence $\psi_{1}\left(y^{(i)}\right)$ is farther than $y^{(i)}$ from $x$ (see Figure 5). 


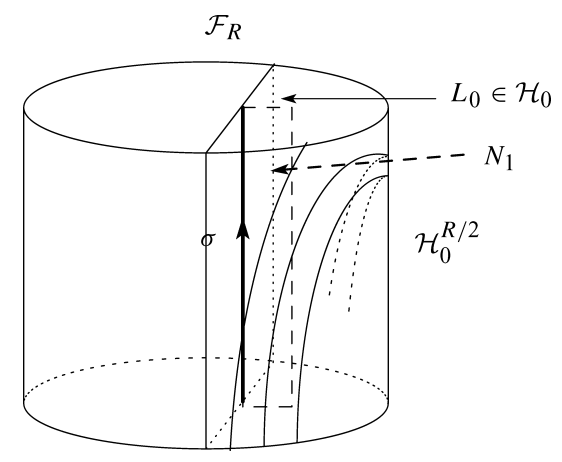

FIGURE 4. The set $N_{1}$.

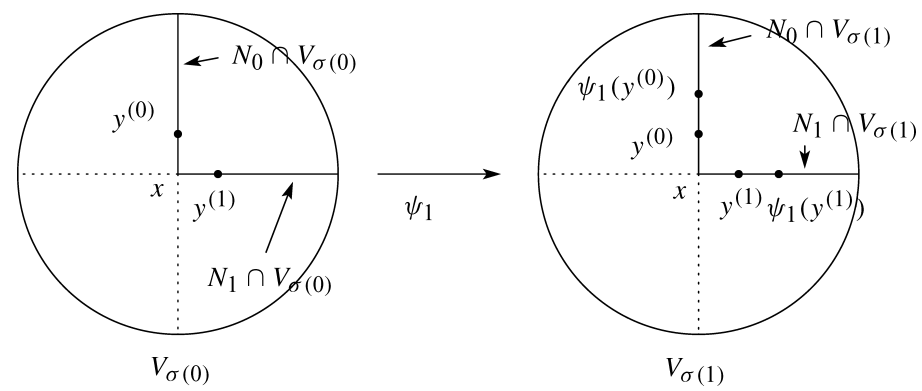

FIGURE 5. The map $\psi_{1}$.

Thus there exists a constant $l_{i}>1$ such that

$$
\mathrm{d} \psi_{1}\left(\dot{\alpha}^{(i)}(0)\right)=l_{i} \dot{\alpha}^{(i)}(0),
$$

which is a contradiction by Lemma 5.5. Therefore there exists no Reeb component consisting of timelike leaves.

Proposition 5.6. The case (3) does not occur, that is, there exists no Reeb component $\left(D^{2} \times S^{1}, \mathcal{F}_{R}\right)$ such that the boundary leaf $\partial\left(D^{2} \times S^{1}\right)$ and at least one of the inner leaves are lightlike.

ProOF. Assume that there is a Reeb component $\left(D^{2} \times S^{1}, \mathcal{F}_{R}\right)$ such that the boundary leaf $\partial\left(D^{2} \times S^{1}\right)$ and one of the inner leaves of $\mathcal{F}_{R}$ are lightlike. Put $F_{1}=\partial\left(D^{2} \times S^{1}\right)$. Let $F_{2}$ be a lightlike leaf of $\left.\mathcal{F}_{R}\right|_{\operatorname{Int}\left(D^{2} \times S^{1}\right)}$. Let $\mathcal{N}_{i}$ be the foliation of $F_{i}$ determined by the lightlike vectors for $i=1,2$. By [14], the one-dimensional foliation determined by the lightlike vectors of a lightlike leaf $F$ is a Riemannian foliation of $F$. Since the foliation $\mathcal{N}_{1}$ is a Riemannian foliation of $F_{1} \cong T^{2}$, we have the following two cases:

(c) All the leaves of $\mathcal{N}_{1}$ are closed.

(d) All the leaves of $\mathcal{N}_{1}$ are dense. 
Let $l$ and $m$ be a longitude and a meridian on $F_{1}$, respectively.

Case 1. There exists a $C^{\infty}$ closed curve $c: S^{1} \rightarrow F_{1}$ which is transverse to $\mathcal{N}_{1}$ and homotopic to $m$. (This case occurs except the case (c) where the homology class of a leaf $L \in \mathcal{N}_{1}$ is equal to $[m]$.)

We extend $c$ to a $C^{\infty}$ embedding $\hat{c}: S^{1} \times[0, \varepsilon) \rightarrow D^{2} \times S^{1}$ which satisfies the following:

(1) The set $\hat{c}\left(S^{1} \times\{t\}\right)$ is on an $\mathcal{F}_{R}$-leaf for all $t \in[0, \varepsilon)$.

(2) $\hat{c}(s, 0)=c(s)$ for all $s \in S^{1}$.

Since the curve $c$ is spacelike, there exists an $\varepsilon_{1} \in(0, \varepsilon]$ such that the curve $\hat{c}\left(\cdot, t_{0}\right)$ is spacelike for all $t_{0} \in\left[0, \varepsilon_{1}\right)$. Since the leaf $F_{2}$ approaches $F_{1}$, there exists a $t_{1} \in\left(0, \varepsilon_{1}\right)$ such that $\hat{c}\left(S^{1} \times\left\{t_{1}\right\}\right) \cap F_{2} \neq \emptyset$. Hence the curve $\hat{c}\left(\cdot, t_{1}\right)$ on $F_{2}$ is transverse to the foliation $\mathcal{N}_{2}$. One of the connected components of $F_{2} \backslash \hat{c}\left(S^{1} \times\left\{t_{1}\right\}\right)$ is a 2-disk, which is a contradiction by the standard Euler class argument.

Case 2. The case (c) where the homology class of a leaf $L \in \mathcal{N}_{1}$ is equal to $[\mathrm{m}]$.

Fix a Riemannian metric $h$ on $D^{2} \times S^{1}$. Let $\mathcal{N}_{i}^{\perp}$ be the foliation of $F_{i}$ perpendicular to $\mathcal{N}_{i}$ with respect to $h$ for $i=1,2$. Fix a leaf $L \in \mathcal{N}_{1}$ and parametrize $L$ by a diffeomorphism $c: S^{1} \rightarrow L \subset F_{1}$. The curve $c$ is transverse to $\mathcal{N}_{1}^{\perp}$. We extend $c$ to a $C^{\infty}$ embedding $\hat{c}: S^{1} \times[0, \varepsilon) \rightarrow D^{2} \times S^{1}$ which satisfies the following:

(1) The set $\hat{c}\left(S^{1} \times\{t\}\right)$ is on an $\mathcal{F}_{R}$-leaf for all $t \in[0, \varepsilon)$.

(2) $\hat{c}(s, 0)=c(s)$ for all $s \in S^{1}$.

Let $X: \hat{c}\left(S^{1} \times[0, \varepsilon)\right) \rightarrow T \mathcal{F}$ be a $C^{\infty}$ non-singular vector field satisfying

$$
\left.X\right|_{\hat{c}(s, t)} \perp \hat{c}_{*}\left(\frac{\partial}{\partial s}\right) \quad \text { for all }(s, t) \in S^{1} \times[0, \varepsilon)
$$

with respect to $h$. The vector field $\left.X\right|_{c\left(S^{1}\right)}$ is spacelike and tangent to $\mathcal{N}_{1}^{\perp}$. So there exists an $\varepsilon_{1} \in(0, \varepsilon]$ such that the vector field $\left.X\right|_{\hat{c}\left(S^{1} \times\left[0, \varepsilon_{1}\right)\right)}$ is spacelike. Since the leaf $F_{2}$ approaches $F_{1}$, there exists a $t_{1} \in\left(0, \varepsilon_{1}\right)$ such that $\hat{c}\left(S^{1} \times\left\{t_{1}\right\}\right) \subset F_{2}$. Since the vector field $\left.X\right|_{\hat{c}\left(S^{1} \times\left\{t_{1}\right\}\right)}$ is spacelike, it is transverse to $\mathcal{N}_{2}$. Hence the orthogonal complement $\left.(\operatorname{Span}\{X\})^{\perp}\right|_{\hat{c}\left(S^{1} \times\left\{t_{1}\right\}\right)}$ in $T F_{2}$ with respect to $h$ is transverse to $\mathcal{N}_{2}^{\perp}$. Therefore the curve $\hat{c}\left(\cdot, t_{1}\right)$ is transverse to $\mathcal{N}_{2}^{\perp}$, which is a contradiction.

PROPOSITION 5.7. The case (4) does not occur, that is, there exists no Reeb component $\left(D^{2} \times S^{1}, \mathcal{F}_{R}\right)$ such that all the inner leaves of $\mathcal{F}_{R}$ are timelike and the boundary is lightlike.

PROOF. By the assumption of time-orientability of $g$, we fix a non-singular, timelike vector field $T \in \Gamma\left(T\left(D^{2} \times S^{1}\right)\right)$. There exist two subfoliations $\mathcal{L}_{0}, \mathcal{L}_{1}$ of $\left.\mathcal{F}_{R}\right|_{\operatorname{Int}\left(D^{2} \times S^{1}\right)}$ by Proposition 4.4. There exists the foliation $\mathcal{N}$ determined by the lightlike vectors on $\partial\left(D^{2} \times\right.$ $\left.S^{1}\right) \in \mathcal{F}_{R}$. Three foliations $\mathcal{L}_{0}, \mathcal{L}_{1}, \mathcal{N}$ are orientable.

LEMMA 5.8. For all point $p \in \partial\left(D^{2} \times S^{1}\right)$, there exists a neighborhood $V_{p}$ around $p$ in $D^{2} \times S^{1}$ and a non-singular, $\mathcal{F}_{R}$-tangent vector field $X^{(p)}$ defined on $V_{p}$ which satisfies the following:

(1) $\left.X^{(p)}\right|_{V_{p} \cap \partial\left(D^{2} \times S^{1}\right)}$ is positive and lightlike. 
(2) $\left.\quad X^{(p)}\right|_{V_{p} \cap \operatorname{Int}\left(D^{2} \times S^{1}\right)}$ is positive and timelike.

Proof. Fix an arbitrary point $p$ in $\partial\left(D^{2} \times S^{1}\right)$. Let $\varphi: U_{p} \rightarrow \boldsymbol{R}^{2} \times[0, \infty)$ be a foliation chart around $p$, where we assume that $\varphi\left(\partial\left(D^{2} \times S^{1}\right) \cap U_{p}\right) \subset \boldsymbol{R}^{2} \times\{0\}$ and that $\varphi^{-1}\left(\boldsymbol{R}^{2} \times\{*\}\right)$ is an $\mathcal{F}_{R}$-plaque. We regard the standard coordinate $\left(x_{1}, x_{2}, x_{3}\right) \in \boldsymbol{R}^{2} \times \boldsymbol{R}$ as a local coordinate of $\boldsymbol{R}^{2} \times[0, \infty)$. Let $\left(g_{i j}\right)$ be the matrix of components of $g$ with respect to $\left(x_{1}, x_{2}, x_{3}\right)$. Fix a positive lightlike vector $v_{p}$ at $p$. We can assume that $v_{p}$ equals $\partial / \partial x_{1}+b(p) \partial / \partial x_{2}$, where $b(p) \in \boldsymbol{R}$ is a constant. We then have

$$
\begin{aligned}
0 & =g\left(\frac{\partial}{\partial x_{1}}+b(p) \frac{\partial}{\partial x_{2}}, \frac{\partial}{\partial x_{1}}+b(p) \frac{\partial}{\partial x_{2}}\right) \\
& =g_{11}(p)+2 b(p) g_{12}(p)+(b(p))^{2} g_{22}(p) .
\end{aligned}
$$

Consider the equation

$$
g_{11}+2 b g_{12}+b^{2} g_{22}=0 .
$$

Since all the leaves of $\mathcal{F}_{R}$ are timelike or lightlike, we have

$$
g_{11} g_{22}-g_{12}^{2} \leq 0 .
$$

Now we will prove that there exists a neighborhood $V_{p} \subset U_{p}$ around $p$ such that the function $g_{22}$ is positive on $V_{p}$. Since $\partial / \partial x_{1}+b(p) \partial / \partial x_{2}$ is lightlike and $g_{11}(p) g_{22}(p)-$ $\left(g_{12}(p)\right)^{2}=0$, we have $g_{22}(p) \neq 0$. Since $\left.g\right|_{T_{p}\left(\partial\left(D^{2} \times S^{1}\right)\right)}$ is positive indefinite, we have

$$
g_{p}\left(\frac{\partial}{\partial x_{2}}, \frac{\partial}{\partial x_{2}}\right)=g_{22}(p) \geq 0 \text {. }
$$

Thus $g_{22}(p)>0$. Therefore there exists a neighborhood $V_{p} \subset U_{p}$ around $p$ such that $\left.g_{22}\right|_{V_{p}}>0$.

Since $g_{22} \neq 0$ on $V_{p}$, we can solve the equation (1) as

$$
b=\left(-g_{12} \pm \sqrt{\left.g_{12}^{2}-g_{11} g_{22}\right)} / g_{22}\right. \text {. }
$$

Define the vector field $X^{(p)}$ defined on $V_{p}$ by

$$
X^{(p)}=\frac{\partial}{\partial x_{1}}+\frac{-g_{12}}{g_{22}} \frac{\partial}{\partial x_{2}} .
$$

This is an $\mathcal{F}_{R}$-tangent, non-singular vector field. We then have

$$
g\left(X^{(p)}, X^{(p)}\right)=\left(g_{11} g_{22}-g_{12}^{2}\right) / g_{22} .
$$

Hence $\left.X^{(p)}\right|_{V_{p} \cap \partial\left(D^{2} \times S^{1}\right)}$ is positive and lightlike, and $\left.X^{(p)}\right|_{V_{p} \cap \operatorname{Int}\left(D^{2} \times S^{1}\right)}$ is positive and timelike.

Fix a collar neighborhood $C: \partial\left(D^{2} \times S^{1}\right) \times[0,1] \rightarrow D^{2} \times S^{1}$, where $C\left(\partial\left(D^{2} \times S^{1}\right) \times\right.$ $\{0\})=\partial\left(D^{2} \times S^{1}\right)$. For all $p \in \partial\left(D^{2} \times S^{1}\right)$, define an open subset $V_{p}^{\prime} \subset \partial\left(D^{2} \times S^{1}\right)$ and a constant $\varepsilon_{p}>0$ such that

$$
p \in V_{p}^{\prime} \subset \partial\left(D^{2} \times S^{1}\right) \cap V_{p}, \quad C\left(V_{p}^{\prime} \times\left[0, \varepsilon_{p}\right)\right) \subset V_{p} .
$$


Put $\hat{V}_{p}=C\left(V_{p}^{\prime} \times\left[0, \varepsilon_{p}\right)\right)$. We have $\bigcup_{p \in \partial\left(D^{2} \times S^{1}\right)} V_{p}^{\prime} \supset \partial\left(D^{2} \times S^{1}\right)$. Hence there exist points $p_{1}, \ldots, p_{k} \in \partial\left(D^{2} \times S^{1}\right)$ such that $\bigcup_{i=1}^{k} V_{p_{i}}^{\prime}=\partial\left(D^{2} \times S^{1}\right)$. Let $\left\{\rho_{i}\right\}_{i=1}^{k}$ be a partition of unity subordinate to the covering $\left\{V_{p_{i}}^{\prime}\right\}_{i=1}^{k}$. We extend $\rho_{i}$ to the function $\hat{\rho}_{i}$ defined on $\hat{V}_{p_{i}}=C\left(V_{p_{i}}^{\prime} \times\left[0, \varepsilon_{p_{i}}\right)\right)$ by

$$
C\left(V_{p_{i}}^{\prime} \times\left[0, \varepsilon_{p_{i}}\right)\right) \stackrel{C^{-1}}{\longrightarrow} V_{p_{i}}^{\prime} \times\left[0, \varepsilon_{p_{i}}\right) \stackrel{\text { prj. }}{\longrightarrow} V_{p_{i}}^{\prime} \stackrel{\rho_{i}}{\longrightarrow}[0,1] .
$$

Put $\varepsilon=\min _{i \in\{1, \ldots, k\}} \varepsilon_{p_{i}}$. Then we have $\varepsilon>0$ and

$$
C\left(\partial\left(D^{2} \times S^{1}\right) \times[0, \varepsilon)\right) \subset \bigcup_{i=1}^{k} \hat{V}_{p_{i}} .
$$

Put $O=C\left(\partial\left(D^{2} \times S^{1}\right) \times[0, \varepsilon)\right)$ and

$$
X=\sum_{i=1}^{k} \hat{\rho}_{i}\left(\left.X^{\left(p_{i}\right)}\right|_{\hat{V}_{p_{i}} \cap O}\right) .
$$

By Proposition 2.4, the vector field $X$ is a non-singular, $\mathcal{F}_{R}$-tangent vector field on $O$ which satisfies the following:

(1) $\left.X\right|_{\partial\left(D^{2} \times S^{1}\right)}$ is positive and lightlike.

(2) $\left.X\right|_{\operatorname{Int}\left(D^{2} \times S^{1}\right) \cap O}$ is positive and timelike.

Now, let $N$ be a non-singular, $\mathcal{L}_{0}$-tangent, positive, lightlike vector field on $\operatorname{Int}\left(D^{2} \times S^{1}\right)$. Put

$$
\begin{aligned}
& U_{1}=C\left(\partial\left(D^{2} \times S^{1}\right) \times[0, \varepsilon)\right), \\
& U_{2}=D^{2} \times S^{1} \backslash C\left(\partial\left(D^{2} \times S^{1}\right) \times[0, \varepsilon / 2]\right) .
\end{aligned}
$$

The family $\left\{U_{1}, U_{2}\right\}$ is an open covering of $D^{2} \times S^{1}$. Let $\left\{\lambda_{1}, \lambda_{2}\right\}$ be a partition of unity subordinate to $\left\{U_{1}, U_{2}\right\}$. Put $\hat{X}=\lambda_{1} X+\lambda_{2} N$. Then the vector field $\hat{X}$ is a non-singular, $\mathcal{F}_{R}$-tangent vector field on $D^{2} \times S^{1}$ which satisfies the following:

(1) $\left.\hat{X}\right|_{\partial\left(D^{2} \times S^{1}\right)}$ is positive and lightlike.

(2) $\left.\hat{X}\right|_{\operatorname{Int}\left(D^{2} \times S^{1}\right)}$ is positive.

Let $\hat{\mathcal{N}}$ be the subfoliation of $\mathcal{F}_{R}$ determined by $\hat{X}$. So we have $\left.\hat{\mathcal{N}}\right|_{\partial\left(D^{2} \times S^{1}\right)}=\mathcal{N}$. By [11], the restriction of any subfoliation of $\mathcal{F}_{R}$ to $\partial\left(D^{2} \times S^{1}\right)$ has Reeb components. So the foliation $\left.\hat{\mathcal{N}}\right|_{\partial\left(D^{2} \times S^{1}\right)}$ has Reeb components. Hence $\mathcal{N}$ has Reeb components. However, $\mathcal{N}$ must be a Riemannian foliation on $\partial\left(D^{2} \times S^{1}\right)$ by [14], which is a contradiction. Therefore there exists no Reeb component $\left(D^{2} \times S^{1}, \mathcal{F}_{R}\right)$ such that all the inner leaves of $\mathcal{F}_{R}$ are timelike and the boundary is lightlike.

We have an easy corollary of Theorem 5.1.

COROLLARY 5.9. Let $(M, g)$ be a closed Lorentzian 3-manifold with finite fundamental group and $\mathcal{F}$ a codimension-1, totally geodesic foliation of $M$. Then $\mathcal{F}$ consists of at least two kinds of leaves among spacelike, timelike, and lightlike ones. 
Proof. By [7], the foliation $\mathcal{F}$ has a Reeb component. Therefore $\mathcal{F}$ consists of at least two kinds of leaves.

\section{REFERENCES}

[1] R. A. Blumenthal and J. J. Hebda, De Rham decomposition theorems for foliated manifolds, Ann. Inst. Fourier 33 (1983), 225-235.

[2] R. A. Blumenthal and J. J. Hebda, Ehresmann connections for foliations, Indiana Univ. Math. J. 33 (1984), 597-611.

[ 3 ] Y. CARrière And E. Ghys, Feuilletages totalement géodésiques, An. Acad. Brasil. Ciênc. 53 (1981), 427432.

[4] Y. Carrière And L. Rozoy, Complétude des Métriques Lorentziennes de $\boldsymbol{T}^{2}$ et Difféomorphismes du Cercle, Bol. Soc. Brasil. Mat. 25 (1994), 223-235.

[ 5 ] E. Ghys, Classification des feuilletages totalement géodésiques de codimension un, Comment. Math. Helv. 58 (1983), 543-572.

[ 6 ] J. Hempel, 3-manifolds, Ann. of Math. Studies 86, Princeton University Press, Princeton, N. J., 1976.

[ 7 ] S. P. NoviKov, Topology of foliations, Trans. Moscow Math. Soc. 14 (1965), 248-278.

[ 8 ] B. O’NeILl, Semi-Riemannian geometry, Academic press, Inc., New York, 1983.

[ 9 ] G. OsHIKIRI, Totally geodesic foliations and Killing fields, Tôhoku Math. J. 35 (1983), 387-392.

[10] G. OsHIKIRI, Totally geodesic foliations and Killing fields, II, Tôhoku Math. J. 38 (1986), 351-356.

[11] I. TAmura AND A. S ATo, On transverse foliations, Inst. Hautes. Études. Sci. Publ. Math. 54 (1981), 205-235.

[12] K. Үокимото, Mutual exclusiveness among spacelike, timelike, and lightlike leaves in totally geodesic foliations of lightlike complete Lorentzian two-dimensional tori, Hokkaido Math. J. 31 (2002), 643-663.

[13] K. Yoкumoto, Some remarks on totally geodesic foliations of Lorentzian manifolds, Ph. D. Thesis, Hokkaido University, 2002.

[14] A. ZeGHIB, Geodesic foliations in Lorentz 3-manifolds, Comment. Math. Helv. 74 (1999), 1-21.

DIVISION OF MATHEMATICS

GRAduATE SCHOOL OF SCIENCE

HOKKAIDO UNIVERSITY

SAPPORO 060-0810

JAPAN

E-mail address: yoku@math.sci.hokudai.ac.jp 